\title{
Coreceptor Dependent Signaling in Individual Primary Resting CD4+ T-cells Mediated by Low Levels of HIV Binding Marta Melar ${ }^{1}$, David Ott ${ }^{2}$ and Thomas J Hope ${ }^{* \neq 1}$
}

Address: ${ }^{1}$ Department of Microbiology and Immunology, University of Illinois at Chicago, Chicago, IL and ${ }^{2}$ Basic Research Program, SAICFrederick, Inc. NCI Frederick, Frederick, Maryland

Email: Thomas J Hope* - thope@northwestern.edu

* Corresponding author $\ddagger$ Presenting author

from 2005 International Meeting of The Institute of Human Virology

Baltimore, USA, 29 August - 2 September 2005

Published: 8 December 2005

Retrovirology 2005, 2(Suppl I):S3 doi:I0.I 186/1742-4690-2-SI-S3

In order to enter into the target cell, HIV requires functional contact with CD4 and CCR5 or CXCR4. The last two are G-protein coupled receptors that when activated with chemokines, or HIV envelope can initiate a wide range of biological responses, including $\mathrm{Ca}^{2+}$ mobilization, cytoskeletal rearrangements and cell migration. To determine the specificity of X4-tropic gp120-mediated signaling through CXCR4, we have chosen a microscopybased approach to observe the response at the level of individual cells providing greater sensitivity. Target cells were able to activate a signaling cascade in response to both monomeric recombinant gp120 and virion-bound trimeric gp 120. $\mathrm{C}^{2+}$ elevation was a direct measurement of CXCR4 engagement because it was dependent on the tropism of the envelope, engagement of $\mathrm{CD} 4$, and sensitive to the CXCR4 antagonist AMD-3100. Signaling required much lower levels of envelope when virion associated. Imaging analysis allowed the correlation of the pattern of virion-mediated $\mathrm{C}^{2+}$ fluxing with the exact number of viral particles bound to cells. This analysis revealed that an average of four virions, and as few as two virions associating with a primary resting $\mathrm{T}$ cell could mediate $\mathrm{C}^{2+}$ mobilization. The ability of several virions to stimulate signaling in primary resting $\mathrm{T}$ cells is physiologically relevant and has important implications for AIDS pathogenesis. Funded in part by DHHS NO1-CO-12400 and RO1AI052051. 